\title{
A Finite Domain CLP Solver on Top of Mercury
}

\author{
Henk Vandecasteele, Bart Demoen and Gerda Janssens \\ K.U.Leuven, departement computerwetenschappen, \\ Celestijnenlaan 200A \\ B-3001 Heverlee \\ \{henk.vandecasteele, bart.demoen, gerda.janssens\}@cs.kuleuven.ac.be
}

\begin{abstract}
In this paper we describe a new implementation of the Finite Domain solver ROPE [8], called MROPE II. This new version was preceded by an implementation on top of Prolog [9] and a version using an early version of Mercury [10]. In the previous implementation Mercury was chosen for its speed, compile-time checking properties and fast reliable development. This previous experiment with Mercury was already a success, still there were some problems: for an efficient execution backtrackable destructive assignment was needed. Later releases of the Mercury system [4] contained such backtrackable destructive assignment and also impure declarations. This was all we needed for a new implementation of ROPE: MROPE II. The performance of this new system, with a very high level implementation, approaches the performance of other well known systems.
\end{abstract}

\section{The Finite Domain CLP System MROPE II}

The constraints handled in our new implementation, can easily be deduced from the Mercury types in the interface of the solver:

A constraint has the following type:

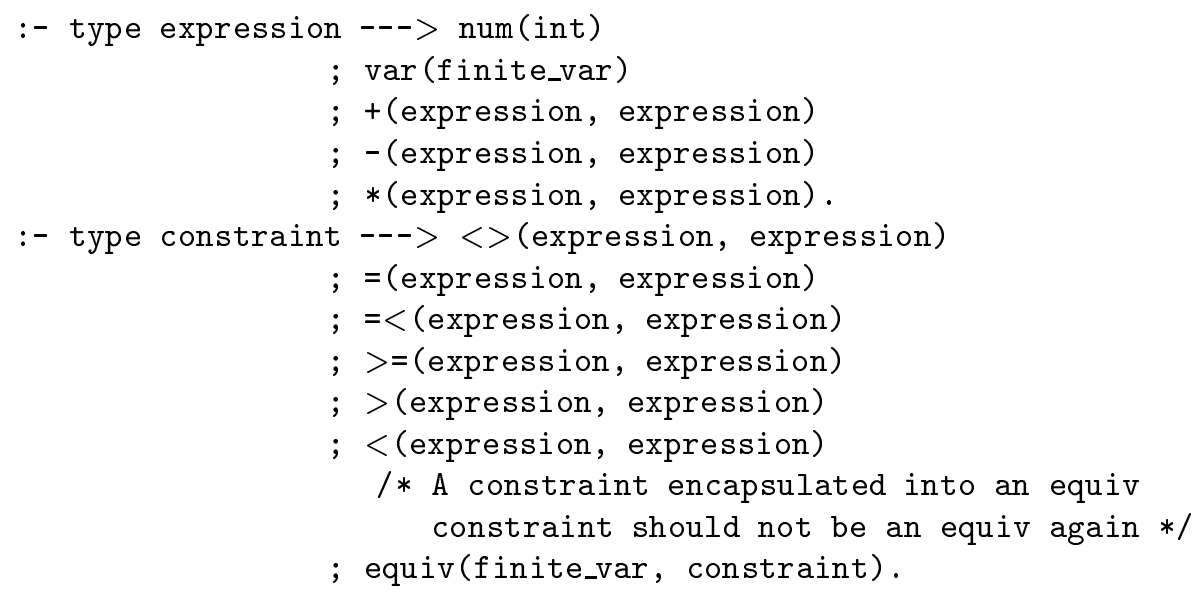

In contrast with the previous solver, indexical constraints are not part of the solver yet. Depending on the need of the applications such constraints can be 
brought into the system very easily. Reification constraints on the other hand are part of the language in the format of equivalence. equiv(finite_var, constraint) is a true constraint if the first parameter is a boolean variable expressing the truth value of the constraint in the second parameter. This second parameter cannot be an equiv/ 2 constraint again. This is not really a restriction on the language and could be overcome with a small transformation. Reification constraints are quite powerful, they can be used as a more flexible stand-in for the cardinality constraint [7].

The type range used to specify a domain of a variable is a bit awkward, but it allows expressing the domains very easily. Also unbounded domains are allowed.

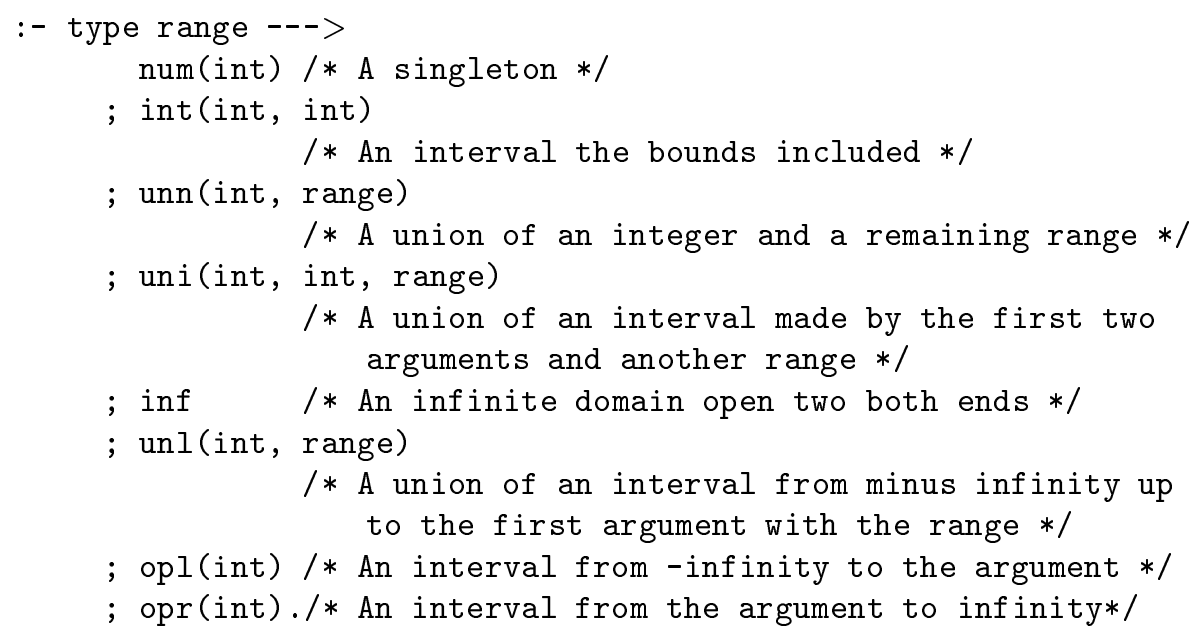

The remainder of the interface defines methods to create new variables, add constraints, find solutions with an enumeration predicate. Also a method is provided to retrieve the current value/domain of Finite Domain variables.

The solver has an explicit current state, containing all finite domain variables and constraints. It has type fd_store. This store is initialised with the predicate init/1.

:- pred init(fd_store).

:- mode init(fd_store_muo) is det.

Every finite domain variable within the system needs to be created before its use. This can be done with the predicate new_var/4.

:- pred new_var(finite_var, range, fd_store, fd_store).

:- mode new_var(out, in, fd_store_mdi, fd_store_muo) is det.

A new constraint is added to the system by calling add_constraint/3.

:- pred add_constraint (constraint, fd_store, fd_store).

:- mode add_constraint(in, fd_store_mdi, fd_store_muo) is semidet. 
Finally enumeration can be started with enum $/ 6$.

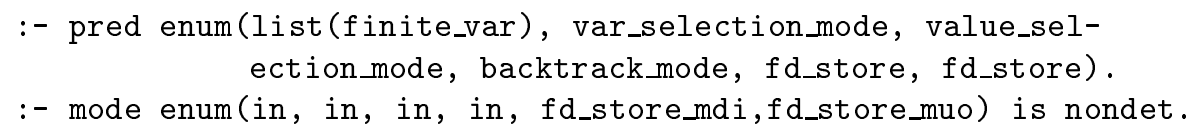

Other variants of the enumeration exist: one in case of optimisation; one with preferred values for the variables; and one combining optimisation and preferred values. An example where enumeration with preferred values is useful is rescheduling.

One can also request for the current domain of a finite domain variable with the predicate value_var.

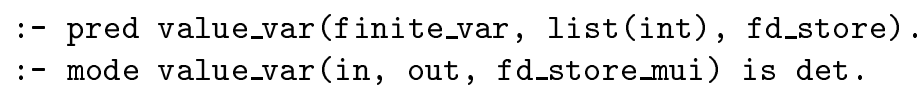

The reader may have noticed that the predicates making up the interface of the solver use the modes fd_store_muo, fd_store_mdi and fd_store_mui. These modes use mostly_unique and mostly_dead instantiation patterns [4]. mostly_unique and mostly_dead allow to specify that there is only one reference to a particular value, and that there will be no more references to that value respectively. Mercury defines some standard modes for manipulating "mostly unique" values:

$\%$ mostly unique output

:- mode muo : : free $->$ mostly_unique.

$\%$ mostly unique input

:- mode mui : : mostly_unique $->$ mostly_unique.

$\%$ mostly destructive input

:- mode mdi : : mostly_unique $->$ mostly_dead.

$f d \_s t o r e \_m d i$ is equivalent to $m d i$ but specific for type $f d \_s t o r e . f d \_s t o r e \_m u o$ relates to muo in the same way and fd_store_mui relates to mui. In our case it means that when a store has been used as a parameter with mode fd_store_mdi, the variable referring to this store can not be used in the remainder of the current predicate. A parameter used in a call as parameter with mode fd_store_muo is known to be the only reference to the current store. The mode fd_store_mui means that the corresponding parameter is the only reference to that data, and the code of the called predicate will keep it that way. An important property of these mostly_unique and mostly_dead instantiation patterns is the possibility to use them in non-deterministic code. This is what mostly stands for. A parameter which is mostly_dead cannot be used in future code, and is therefore dead, but it could come alive again after backtracking. An example of a program using this MROPE II module can be found in Section 4. 


\section{A Log of Previous Experiments}

\subsection{Implementing a Finite Domain Solver in Prolog}

In Logic Programming, it is standard practice to implement enhanced Logic Programming languages in Prolog through meta-interpretation. The advantage of such an approach is that those features of the enhanced language that coincide with corresponding features of Prolog can be implemented through downward reflection. Only features of the language that require a treatment different from that in Prolog are reified in the meta-interpreter. However, it is well-known that this type of implementation may produce significant overhead. One of the main motivations of LP work on partial evaluation has been to remove this overhead through transformation. We refer to [1] for a more extensive discussion on these issues.

It has frequently been observed that an alternative solution, avoiding the need for partial evaluation, is to implement the enhanced language by means of a transformer which maps the enhanced language to Prolog. Denoting a metainterpreter for the enhanced language as $\mathrm{M}$ and a partial evaluator for Prolog as $\mathrm{PE}$, conceptually such a transformer can be defined as a program $\mathrm{T}$, such that for every program $P$ in the enhanced language: $T(P) \equiv P E(M(P))$. The point raised in discussions on this topic is that writing $\mathrm{T}$ from scratch is often not significantly more difficult than writing $\mathrm{M}$ and should therefore be considered as a valid (and more economic) approach to implementing the enhanced language.

In this version of the ROPE language such a "transformation approach" was taken. The Finite Domain CLP program is then transformed to Prolog program which contains calls to a Finite Domain Library.

Passing Information Around In case there is no extra information to be passed around such a transformation $\mathrm{T}$ is quite simple: wrapping the special features of the enhanced language in calls to predefined library predicates will do the job. Given the program:

Example 1.

$a(X, Y):-r(X), c(X, Y)$.

$\mathrm{a}(\mathrm{X}, \mathrm{Y}):-\mathrm{s}(\mathrm{X}, \mathrm{Y}, \mathrm{Z}), \mathrm{a}(\mathrm{Y}, \mathrm{Z})$.

with $r / 1$ and $s / 3$ special calls.

Then this would be transformed to:

Example 2.

$\mathrm{a}(\mathrm{X}, \mathrm{Y})$ : - takeCare0fFeature $(\mathrm{r}(\mathrm{X})), \mathrm{c}(\mathrm{X}, \mathrm{Y})$.

$\mathrm{a}(\mathrm{X}, \mathrm{Y})$ :- takeCareOfFeature $(\mathrm{s}(\mathrm{X}, \mathrm{Y}, \mathrm{Z})), \mathrm{a}(\mathrm{Y}, \mathrm{Z})$.

If there is need for extra information to be passed around from one call to takeCareOfFeature to another then every clause of the program needs an extra parameter, and the clause must be renamed. For every predicate definition in the program a new clause is added with the original name of the clause which calls the transformed clause with the initialised extra parameters. Applying this technique to the example results in: 
Example 3.

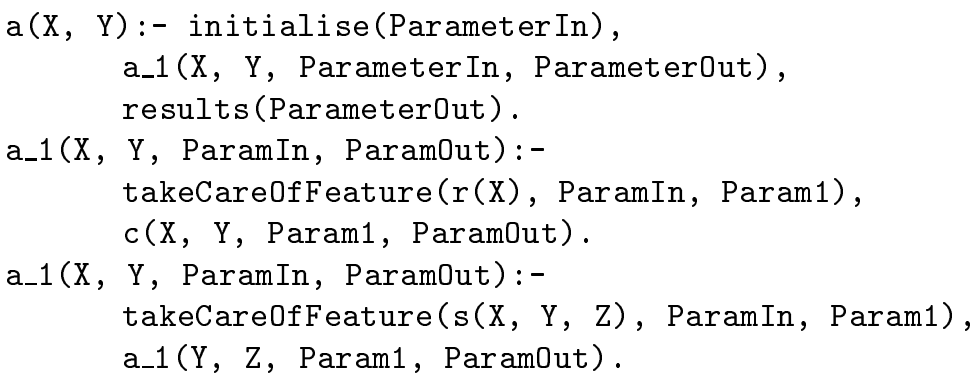

In some cases such parameter passing can be avoided by instantiating the variables on which the special features of the language act, with the information to be passed. This technique is not general but is applicable in our case. Then we also have to rename the predicates, as we have to intercept the output of the program and reconstruct the output that the original program was supposed to produce. The compiled program then looks like:

Example 4.

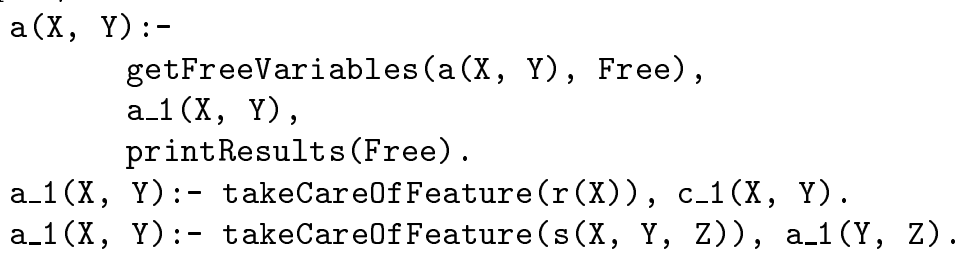

First the free variables are extracted from the query. After successful completion of the program we print the results of the original program. The user is responsible for preventing Prolog from printing the internal representation of $\mathrm{X}$ and $\mathrm{Y}$. This can be done by adding a fail to the query: $a(X, Y), !$, fail in case only one solution is needed, $a(X, Y)$, fail if all solution are wanted.

Of course with this kind of technique special care must be taken. Normal Prolog unification of the special variables must be prevented while transforming the program. Also the use of builtins on these special variables must be taken care of.

The Finite Domain Library Before execution of such programs above a library must be provided. More specific for a finite domain constraint solver a predicate must be defined to handle a constraint to replace to calls to takeCareOfFeature/1. There are three predicates in the finite domain library:

- getFreeVariables/2. This predicate searches for the free variables in first arguments and stores them in a list in the second argument.

- printResults/1 prints out the list of Prolog expressions while substituting the finite domain variable with their semantic value. 
- takeCareOfFeature/1 (constraint_-/1 in our case) is by far the most interesting predicate of the finite domain library. This predicate instantiates domain variables to a specific structure, fulfilling the purpose of passing on relevant information for the solver, adds the constraint to the constraint store and activates the solver for checking the constraint.

In the next subsections we explain how the store, containing constraints and domains, is kept.

Representation of the Store Within the constraint solver constraints and domains of Finite Domain variables must be stored in some way. These domains and constraints should be easily accessible. Domains are updated and used very frequently. Whenever a domain changes, constraints affected must be activated. As stated before, one alternative is to add to every clause an extra input and output parameter. The data structures in these extra parameters contain information on the constraints connected to the variables and the domains as well. Every finite domain variable then refers to this global data structure with a unique number. When information concerning a finite domain variable is changed, for example its domain becomes smaller, then the out parameter reflects these changes while it still contains the old information on the other variables. Such a working method results in efficiency problems as updates to this global store are dependent on the number of finite domain variables in the system. An example of such a data structure is a flat term where each argument contains information on one finite domain variable (in the sequel, we refer to this representation as "functor"). The unique number associated to each finite domain variable is the position of this argument in the functor. If the information on one variable changes then a new functor must be created where all arguments but one must be copied from the old functor. Time and place complexity of this operation is $\mathrm{O}(\mathrm{N})$, where $\mathrm{N}$ is the number of finite domain variables in the program. A better alternative is a tree structure. In this case the complexity of copying in case of changes is logarithmic in the number of existing finite domain variables. Unfortunately, also access without modification becomes logarithmic in the number of finite domain variables.

Therefore we choose to instantiate each finite domain variable to a structure that contains both the domain of the variable and the constraints in which this variable is involved. Then extra arguments for every clause in the program containing information on the current state of constraints and domains are not needed. The access to the data on one variable, having the variable available, becomes independent of the number of variables. There exist several references to one finite domain variable, namely each constraint in which the variable occurs. As a result we cannot replace the finite domain variable with a new finite domain variable when its domain changes. For this purpose we will use open ended data structures ${ }^{1}$. Passing on information on constraints and domains through the domain variable itself is particularly interesting for our application, since we

\footnotetext{
${ }^{1}$ we come back later to this matter, together with the time complexity issue involved.
} 
need to propagate constraints as soon as some type of changes occurs to the domain of a variable. Thus, by instantiating a domain variable to a structure finiteVar(Domain, Constraint_Store), where Domain is a representation of the domain of the variable and Constraint_Store of all constraints in the store that contain the variable, easy access for propagation is guaranteed. Actually there are only four classes of constraints to be activated.

- Constraints to be activated after every change to the domain of a variable.

- Constraints to be activated after a variable becomes ground.

- Constraints to be activated after the lower bound of a variable's domain changes.

- Constraints to be activated after the upper bound of a variable's domain changes.

Note that some constraints belong to more than one class. The structure for the constraint store is then easily chosen: store(Always, Ground, LowerBound, UpperBound). With Always the constraints that need to be checked whenever the domain of the associated variable changes, Ground the constraints that need to be checked as soon as the finite domain variable becomes ground and Lowerbound and UpperBound whenever the lower bound (resp the upper bound) of the domain changes. If we handle a new constraint we check for the operators used in the expression. An expression $\operatorname{int}(X), \min (X)$ will lead to storing the constraint in the LowerBound constraint list of the variable X. $\operatorname{dom}(Y)$ tells the system to put the constraint in the Always constraint list of variable Y. ask(ground( $Z)$, Constraint) will put the constraint in the Ground list of the variable Z.

Suppose we have a small program:

\section{Example 5.}

\section{/*}

suppose 9 animals are playing on the grass, Rabbits and Pheasants, 24 feet can be seen, how many animals of each kind are there ? */ rabbits $(R, P):-$

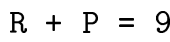

In this approach these 2 constraints will be transformed to low-level constraints: $\left\{\mathrm{P}\right.$ in $9-\operatorname{int}(\mathrm{R}), \mathrm{R}$ in $9-\operatorname{int}(\mathrm{P}), \mathrm{P}$ in $\left(24-4^{*} \operatorname{int}(\mathrm{R})\right) \operatorname{div} 2, \mathrm{R}$ in $(24-$ $2 * \operatorname{int}(\mathrm{P})) \operatorname{div} 4\}^{2}$.

After treating the 4 low-level constraints of the rabbit program we obtain the following instantiation of the finite domain variables $\mathrm{P}$ and $\mathrm{R}$ :

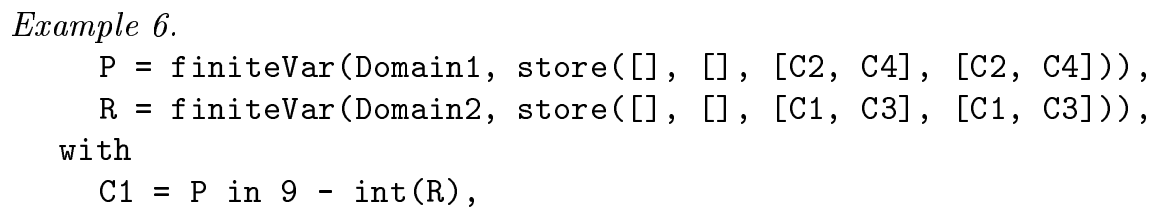

$\overline{2}$ int(R) means: the smallest interval including the domain variable $R$ 


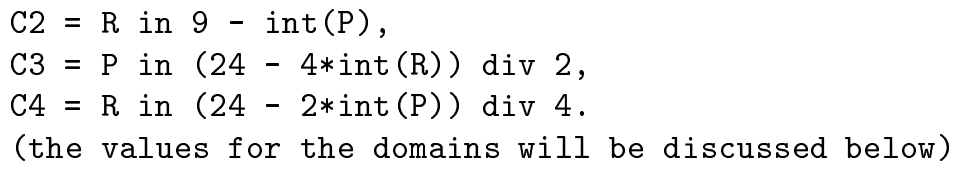

These data structures are generated while the predicate constraint_/1 from the finite domain library handles a new constraint. Note that the representation we choose here for the list of constraints is too simplistic. Since constraints can be added at any time of the execution we have to use open ended data structures, discussed below.

Representation of the Domains As we are going to reason on the bounds of the domains, add and subtract domains we need a representation which is convenient for this kind of computations. Therefore we choose a union of intervals. For example a domain with the values $1,2,3,4,8,10,11,12$ will be represented as $1 . .4 \vee 8 . .8 \vee 10 . .12$ using the same operators as defined in the low-level language. A fresh finite domain variable is initialised with the domain 0. .infinity. As we have to update the domains of the finite domain variables we also need a structure which we can update by further instantiating. Using an open ended list for explaining the principle, every finite domain variable then starts with the domain representation: [0..infinity| -]

For the rabbit problem: after adding the two constraints constraint1 and constraint2 both finite domain variables are instantiated as

finiteVar([0..infinity, $0 . .9 \mid$ _], Constraint_Store).

after adding the two other constraints constraint 3 and constraint4 the data structure becomes

$$
\begin{aligned}
& \mathrm{P}=\text { finiteVar}([0 . \text { infinity, } 0 . .9,3 . .7,5 . .6,6 . .6 \mid-], \text { Store1 }) \\
& \mathrm{R}=\text { finiteVar( }([0 . \text { infinity, } 0 . .9,2 . .6,3.4,3.3 \mid-], \text { Store2 })
\end{aligned}
$$

As a result of adding the two constraints to the constraint store and letting them propagate the two variables become ground. How this result is achieved is explained below.

Open Ended Data Structures This subject has already been mentioned several times above: the part of the store connected to one variable cannot be replaced but should always be changed by further instantiating it. In case of the constraints a simple open ended list can be used. When adding a new constraint the end of the list is further instantiated with a new element, the new constraint, and a fresh variable becomes the new end of the list. Also, for some optimisations in the finite domain library and for implementing constraints like cardinality, there is need for removing constraints from the constraint store. This is solved by adding a free variable to every constraint. A constraint can be deactivated by instantiating this free variable to the atom 'old'. When activating constraints, after a domain of a finite domain variable has changed, such constraints must be skipped. Similar to adding new constraints to the store, the domain of the Finite Domain variable should be changed during computation. In principle this could 
again be done with an open ended list. The annoying thing here is that access to the domain, and updating as well, becomes linear in the number of updates to the finite domain variable. Indeed when fetching the current domain, the whole list of old domains must be traversed until the last element before the open end is reached. In our implementation we used an open ended data structure based on trees, which we will call open ended trees. The access and update complexity of this open ended tree is logarithmic in the number of updates to the domain. Moreover an extra variable is reserved which is instantiated when the domain becomes a singleton, hence fetching the domain of a Finite Domain variable which has been assigned can be done in constant time. For the curious reader interested in this topic the code handling this feature is added:

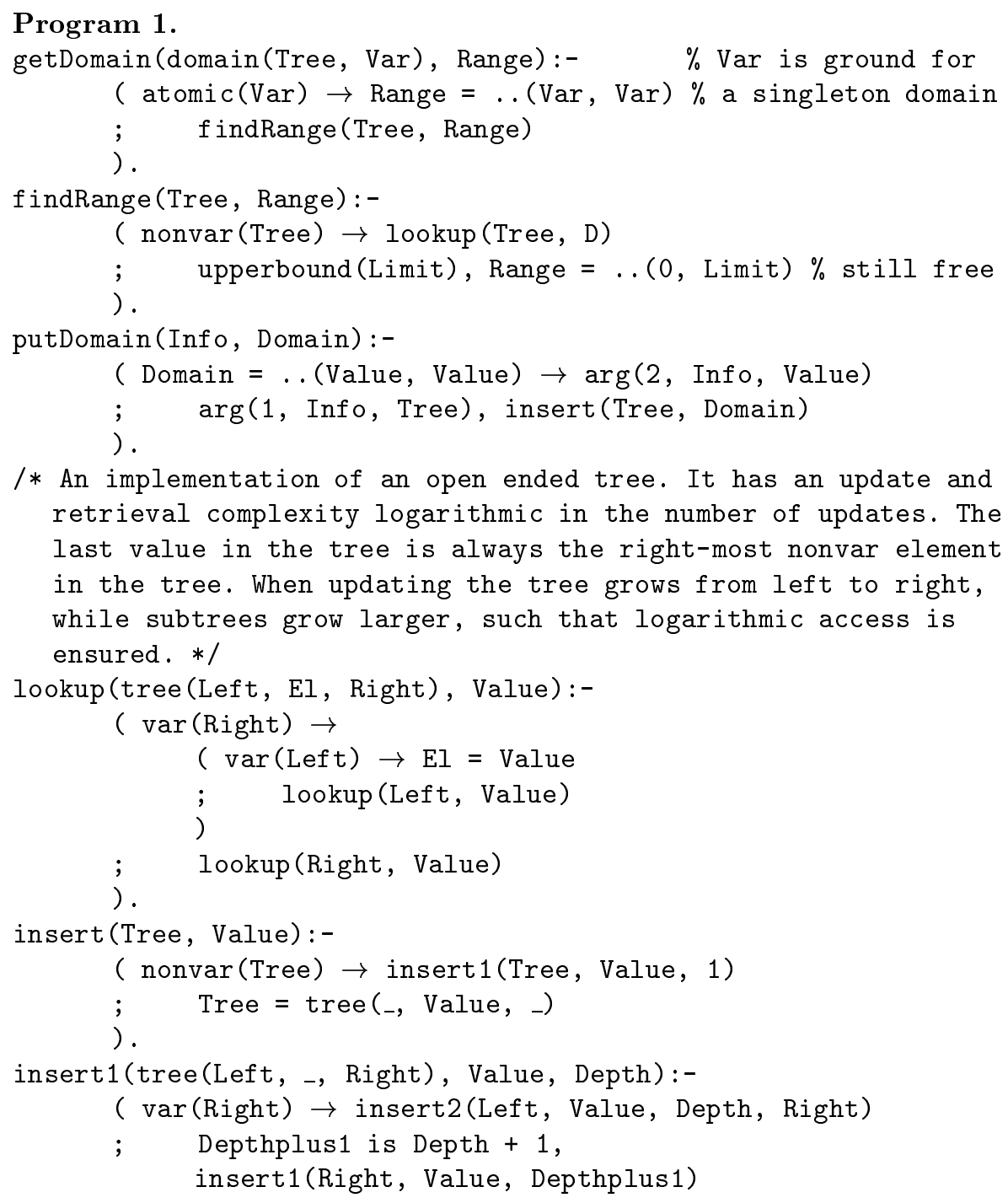




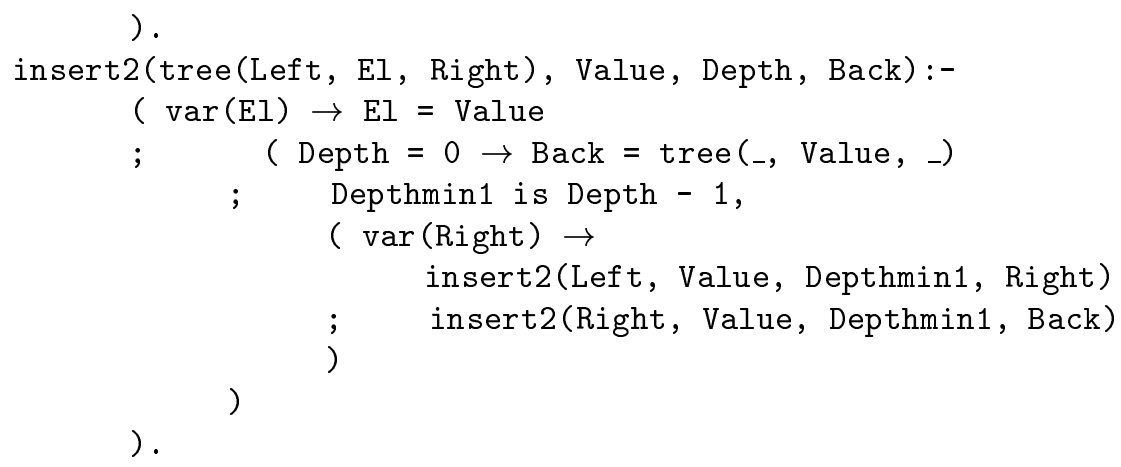

Queue of Constraints A last data structure concerns the queue of constraints which were activated, but not used yet. This is an open ended list where new constraints are added at the end of the list. A desirable feature of such a queue is that a constraint can only appear once in the queue. For this purpose an unbalanced binary tree is used in the pure Prolog version. In an impure version, we have used a record database. Every constraint has a unique number. For every constraint in the queue this number is stored in the tree/database.

\subsection{Early Mercury as a Platform for a Solver}

At the time of this first experiment, Mercury [5] was a recent phenomenon in the field of logic programming: it was faster than other logic language implementations (e.g. Prolog) and better suited for the development of large applications because of its compile time error detection capabilities. The implementation described in the previous subsection is a prototype implemented in pure Prolog [9]. This implementation will further be referred to as ROPE. This prototype lacks efficiency because its implementation doesn't rely on any non-standard support of the Prolog implementation. On the other hand, this helped the development of the ideas of ROPE quite a bit. We believe that the reason for this inefficiency is partly the generality of Prolog (reversible, non-typed predicates) and partly the lack of support for data structures that can be updated at constant cost. When the new logic programming language Mercury emerged, it looked very promising to port our prototype to this new system. One of the advantages of Mercury is faster execution: type, mode and determinacy declarations allow to generate more efficient code. In this subsection we summarise the work presented in [10] and [11].

A Preview on Efficiency Gain As an initial experiment we ported the code computing intersections of domains. Then we compared the speed of the Prolog version on ProLog_by_BIM and the Mercury version on a Sparccenter-1000.

About 40.000 intersections were computed.

The table 1 shows that it is reasonable to expect a 10-fold speedup when going from Prolog to Mercury, on the assumption that Mercury offers efficient pure alternatives for the tricks in the Prolog program. 


\begin{tabular}{|r|rr|}
\hline \hline & ProLog_by_BIM Mercury \\
\hline 40.000 intersections & $3.7 \mathrm{~s}$ & $0.36 \mathrm{~s}$ \\
\hline \hline
\end{tabular}

Replacing Open Ended Data Structures Replacing the open ended datastructures, heavily used in the Prolog version, was a hard job. Without using the C-interface for implementing other data-structures is was unavoidable to add extra parameters to the program passing information around about the state of the finite domain variables and the involved constraints. For representing this global state a Mercury array was used. To our surprise the program was slower than the Prolog version. In an attempt to find the reason for this inefficiency, different data-structures were tried in this global store: the arrays were replaced by binary trees and a plain functor.

It turned out that this data-structure was significant as can be seen in table 2

\begin{tabular}{|l|r|}
\hline \hline & queens $(10)$ \\
\hline Mercury array & $47 \mathrm{~s}$ \\
bintree & $25 \mathrm{~s}$ \\
functor $/ 20$ & $21 \mathrm{~s}$ \\
\hline \hline
\end{tabular}

Table 2. Changing the data-structure

The execution time of the Prolog version being 39 seconds, the two new versions were faster than the Prolog execution.

Using Backtrackable Destructive Assignment Finally we decided to hack the Mercury system and add our own backtrackable destructive assignment. Then we could change the data on finite domain variables in-place, having a constant time access and update time-complexity. Next to this in-place modification, we could also add a cardinality constraint to the system. With the additional cardinality constraints a wider range of examples was possible.

For comparison the same code was executed in SICStus v2.1 using setarg/3 because the ROPE system on ProLog_by_BIM and the implementation in Mercury now use totally different data structures.

Table 3 shows a relatively bad result on the queens problem for the MROPE system. In the version of ROPE in Prolog the different from constraint is handled at a higher level, which allows some optimisation: a constraint $\mathrm{X}<>\mathrm{Y}$ can be removed from the constraint store as soon as one variable is instantiated. This was not done in the Mercury version. 


\begin{tabular}{|l|rrrr|}
\hline \hline & MROPE(Mercury) MROPE(SICSTUS) ROPE(BIM) pure MROPE \\
\hline queens & $21 \mathrm{~s}$ & $109 \mathrm{~s}$ & $39 \mathrm{~s}$ & $65 \mathrm{~s}$ \\
bridge1 & $1.9 \mathrm{~s}$ & $10 \mathrm{~s}$ & $27 \mathrm{~s}$ & $5 \mathrm{~s}$ \\
bridge2 & $1.7 \mathrm{~s}$ & $11 \mathrm{~s}$ & $14 \mathrm{~s}$ & \\
perfect & $23 \mathrm{~s}$ & $125 \mathrm{~s}$ & $126 \mathrm{~s}$ & \\
suudoku & $1.2 \mathrm{~s}$ & $5.5 \mathrm{~s}$ & $6.8 \mathrm{~s}$ & \\
\hline \hline
\end{tabular}

Table 3. The effect of backtrackable destructive assignment

So, ignoring the queens result, we observe that MROPE is 5 to 15 times faster than the original ROPE in Prolog.

\section{Using a More Mature Mercury}

As the Mercury system is a constantly evolving system, the Mercury system has become a lot more mature since the experiment reported in the previous section. In the current version (0.8.1) building stones for backtrackable destructive assignment and other features, like impure declarations, were included. This makes it possible to create an efficient but still high level implementation of our Finite Domain solver.

\subsection{Data Structures and Implementation}

A finite domains solver has three main data structures:

- domains attached to the finite domain variables,

- constraints and

- a queue of constraints to be checked in the fixpoint algorithm.

For all operations on these data structures: creation, retrieval and update, it is important to achieve constant time operations. In this section we will show that Mercury allows to define and use such data structure with a minimum of lowlevel programming. The low-level programming concerns the use of backtrackable destructive assignment. A small module mutable defines such operations:

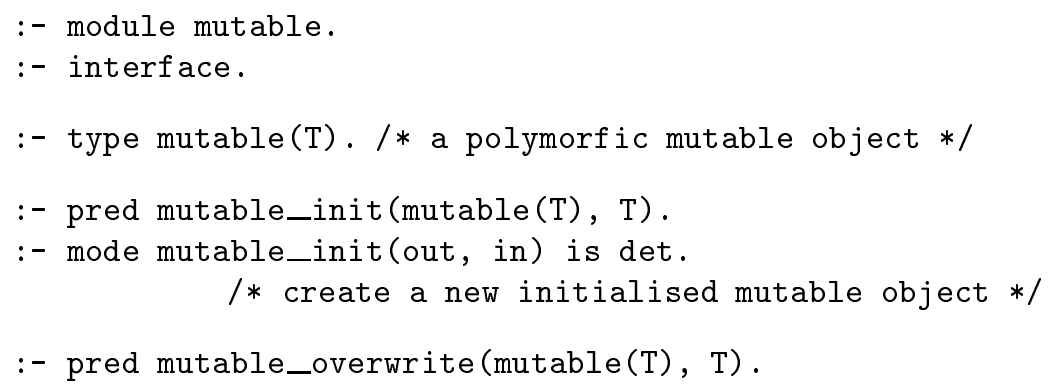




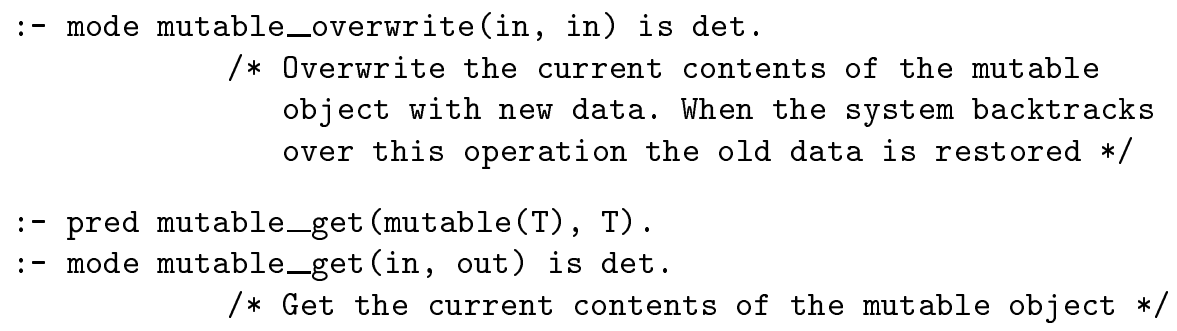

These operations are implemented using the C-interface of the mercury system:

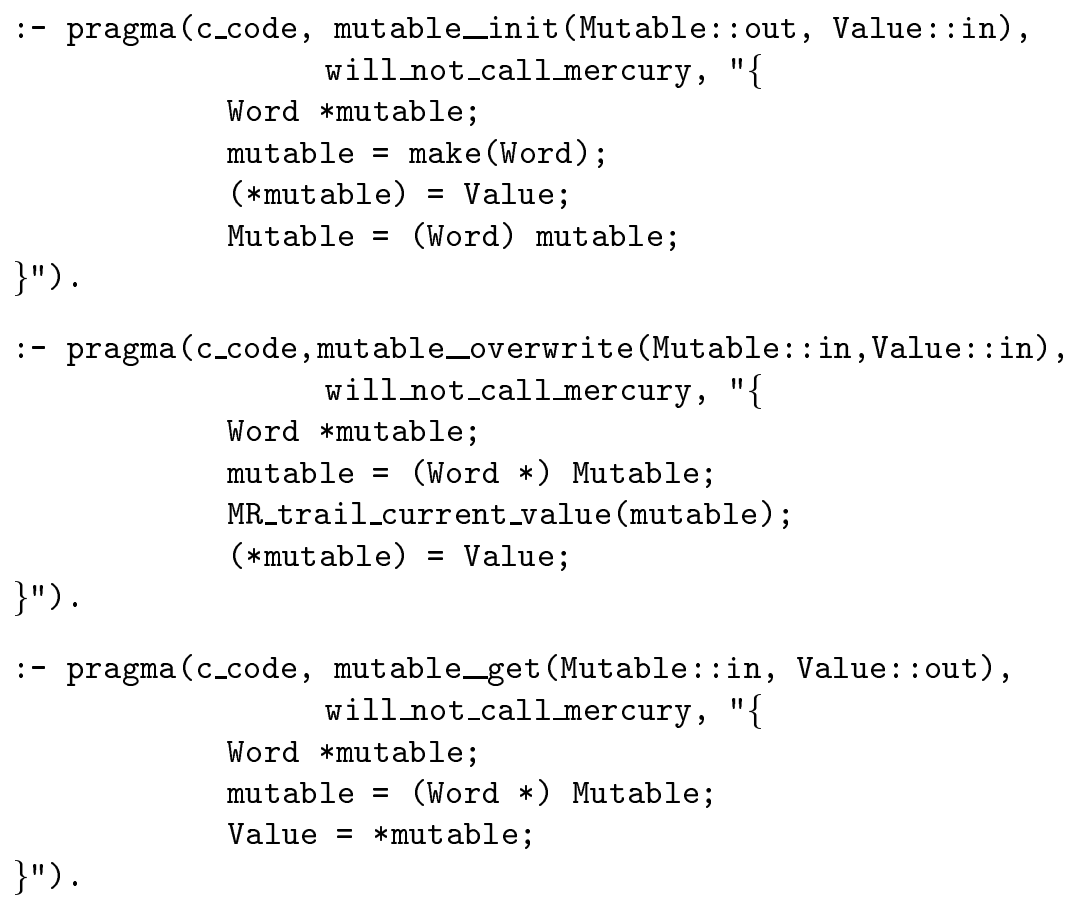

Using the mutable object a whole new range of new data structures can be used: doubly linked lists, difference lists, ... . On creation of a new finite domain variable, the implementation creates a number of slots containing mutable objects.

- A slot for the current domain.

- Five slots for lists of constraints. Each of those five slots contain constraints with a different propagation scheme. The constraints in the first slot are only propagated whenever the domain of the variable becomes a singleton, the second list of constraints becomes active when the lower-bound of the domain changes, the third when the upper bound changes. A fourth list of 
constraints is activated when the upper bound or the lower bound changes ${ }^{3}$. The last list of constraints is propagated whatever change to the domain is made.

- An extra slot can be added containing a string, describing a problem specific meaning of the finite domain variable which can be used for debugging or output purposes.

Since the information in those slots will be updated by backtrackable destructive assignment, there is need to store these slots in a global data structure. When storing them directly as the contents of the finite domain variable, the information will be visible and update-able from anywhere in the program at constant cost. For storing the constraints in one of these five slots holding constraints a doubly linked list is used. This way new constraints can be added, old constraints deleted and constraints retrieved at constant time. The queue of constraints maintained by the fixpoint algorithm is a difference list. The only way to implement a difference list in the current version of Mercury is using backtrackable destructive assignment. This way constraints can be added and removed in constant time. An important property is the ability to concatenate queues in constant time. Of course special attention has to be paid when using these mutable data structures, as bugs in these parts of the program will never be found by the compiler. Fortunately the size of the non-declarative code is rather small, and the advantage of using Mercury is retained for the remainder of the code.

\subsection{Comparison}

A small comparison was made between this new implementation (MROPE II), the clp(fd) library [2] from SICStus, the old implementation of ROPE in Prolog on top of SICStus, GNU prolog [3] and CHIP, one of the leading commercial products in the area having a low-level implementation.

\begin{tabular}{|l|r|r|r|r|r|}
\hline \hline \multicolumn{1}{|c|}{ benchmark } & MROPE II & SICStus & ROPE(SICStus) & CHIP & $\begin{array}{r}\text { GNU Prolog } \\
1.1 .2\end{array}$ \\
\hline queens 8 (all solutions) & 0.12 & 0.25 & 1.06 & 0.08 & 0.04 \\
queens 25 (first solution) & 5.37 & 13.45 & 45.27 & 2.77 & 0.80 \\
bridge1 & 3.35 & 1.96 & 8.79 & 0.82 & 0.18 \\
bridge2 & 2.03 & 2.19 & 4.44 & 1.13 & 0.30 \\
suudoku & 0.31 & 0.16 & 0.2 & 0.18 & 0.07 \\
\hline \hline
\end{tabular}

Table 4. Comparing the solver in Mercury with other systems on a SparcII

\footnotetext{
${ }^{3}$ In previous versions this slot was not available, all constraints to be activated as soon as the lower bound or upper bound changed were added twice to the constraint store for the variable. Once in the list to be activated when the lower bound changed, and once to the list to be activated when the upper bound changed.
} 
Five small benchmarks were selected. The classic queens problem, finding all solutions for queens(8) and finding the first solution for queens(25). Two implementation of the bridge problem [6], the version bridge1 backtracks over the disjunctions for finding the optimal solution, the version bridge? first expresses the disjunctions connecting a boolean variable to each of the disjunctions. In the end the boolean variables are enumerated for finding the optimal solution. In principle the last version should be superior, since the disjunctive constraints are brought into the constraint store while delaying the choice. These constraints can start pruning before they are decided on. The last benchmark is suudoku, a Japanese puzzle.

The benchmarks were performed on both a Solaris machine with a SparcII processor running at $167 \mathrm{Mhz}$ and a Linux machine having a Pentium II 233Mhz. On the Solaris machine the SICStus compiler generates "native code", on the Linux machine the SICStus compiler generates "bytecode", this explains the slower execution on the Linux box. From table 4 we can see the Mercury implementation of our solver approaches the timing of the solver [2] delivered with SICStus. The Mercury implementation outperforms the Prolog implementation ROPE with grace. Our system is still two or three times slower than CHIP, and of course does not have the global constraints of CHIP. The results of running the same benchmarks on Linux, are shown in Table 5. Here the Mercury implementation is superior to the SICStus implementation, because the latter one is using "bytecode" on Linux. In contrast our system is outperformed by GNU Prolog. This shows that compiling the finite domain constraints [3] pays off. Still we can see that running the last benchmark (suudoku) on Linux nearly gets to the speed of GNU Prolog. In this example some preprocessing is done; during that preprocessing we benefit from using Mercury.

\begin{tabular}{|l|r|r|r|r|}
\hline \hline \multicolumn{1}{|c|}{ benchmark } & MROPE II & SICStus & ROPE(SICStus) & GNU Prolog 1.0.0 \\
\hline queens 8 (all solutions) & 0.05 & 0.42 & 1.79 & 0.03 \\
queens 25 (first solution) & 2.7 & 15.7 & 78.69 & 0.9 \\
bridge1 & 1.57 & 2.57 & 14.11 & 0.12 \\
bridge2 & 0.85 & 1.73 & 7.79 & 0.21 \\
suudoku & 0.06 & 0.18 & 0.35 & 0.05 \\
\hline \hline
\end{tabular}

Table 5. Comparing the solver in Mercury with other systems on a Pentium II 


\section{An Example Using MROPE II}

Here an example using the primitives above. It is the implementation of the classic queens program.

\section{Program 2.}

:- pred queens(io_state: :di, io_-state: :uo) is det.

queens $->>$

io_write_string("number of queens?"), $\{$ non_logical_io_read_int (NQ) $\}$,

$(\{$

$$
\text { init (Store1), }
$$




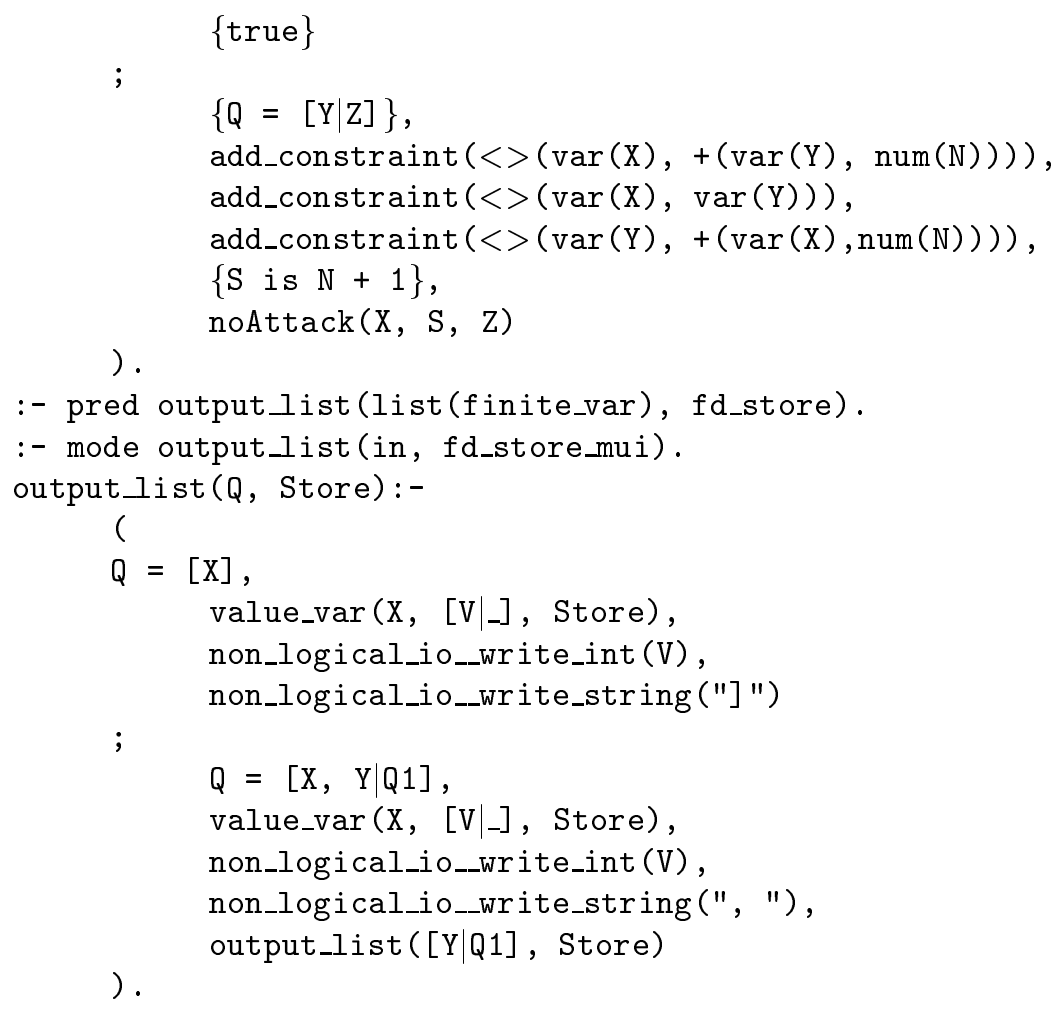

\section{Contribution}

In this paper we show how a Finite Domain solver written on top of Mercury, with very little low-level programming, can be competitive with other well-known systems.

The system is available from

http://www.cs.kuleuven.ac.be/ ${ }^{\sim}$ henkv/mropeII0.1.tar.gz

\section{References}

1. M. Bruynooghe and D. De Schreye. Meta-interpretation. In S. C. Shapiro, editor, Encyclopedia of Artificial Intelligence, pages 939-940. John Wiley \& Sons, Inc, 1992.

2. M. Carlsson, G. Ottosson, and B Carlson. An open-ended finite domain constraint solver. In Proc. of Programming Languages: Implementations, Logics, and Programs, 1997.

3. P. Codognet and D. Diaz. Compiling constraints in $\operatorname{clp}(\mathrm{fd})$. The Journal of Logic Programming, 27(3):185-226, June 1996.

4. Fergus Henderson, Thomas Conway, Zoltan Somogyi, and David Jeffery. The Mercury Language Reference Manual 0.8.1. Melbourne, 1999. 
5. Z. Somogyi, F. Henderson, and T. Conway. The implemenatation of mercury: an efficient declarative logic programming language. In Proceedings of the ILPS'94 Postconference Workshop on Implementation Techniques for Logic Programming Languages, 1994.

6. P. Van Hentenryck. Constraint Satisfaction in Logic Programming. The MIT press, 1989.

7. P. Van Hentenryck and Y. Deville. The cardinality operator: A new logical connective for constraint logic programming. In proceedings of ICLP, pages $745-759$, 1991.

8. H. Vandecasteele. Constraint Logic Programming: Applications and Implementation. PhD thesis, Department of Computer Science, K.U.Leuven, 1999.

9. H. Vandecasteele and D. De Schreye. Implementing a finite-domain CLP-language on top of Prolog : a transformational approach. In Frank Pfenning, editor, Proceedings of Logic Programming and Automated Reasoning, number 822 in Lecture Notes in Artificial Intelligence, pages 84-98. Springer-Verlag, 1994.

10. H. Vandecasteele, B. Demoen, and J. Van Der Auwera. The use of Mercury for the implementation of a finite domain solver. In Proceedings of JICSLP'96 Postconference Workshop on Parallelism and Implementation Technologies for (Constraint) Logic Languages, 1996.

11. H. Vandecasteele, B. Demoen, and J. Van Der Auwera. The use of Mercury for the implementation of a finite domain solver. In I. de Castro Dutra, M. Carro, V. Santos Costa, G. Gupta, E. Pontellia, and Silva F, editors, Nova Science Special Volume on Parallelism and Implementation of Logic and Constraint Logic Programming. Nova Science Publishers Inc, 1999. 